\title{
Influencia de los factores personales y académicos sobre la deserción de estudiantes en la educación a distancia
}

Ana Ilva Capera Urrego ${ }^{1}$

\section{Resumen}

Objetivo. Determinar los elementos que, de acuerdo con la opinión de estudiantes activos y desertores de esta institución, son causa del problema de abandono de los cursos.

Método. La investigación realizada tuvo un enfoque cuantitativo de tipo descriptivo-inferencial, y permitió obtener mediante la aplicación de un cuestionario algunos datos sobre las actitudes, tendencias, percepciones y opiniones de los participantes, respecto a la influencia de los factores personales y académicos, entre otros, que los motivan a permanecer o a desertar de los cursos en los cuales se han matriculado.

Resultados. Los principales factores asociados a deserción fueron: sentimiento de abandono por parte de los tutores, factores académicos, orientación académica, aspectos económicos y responsabilidades familiares, flexibilidad de espacio y tiempo, entre otros.

Palabras clave: Educación a distancia, aprendizaje en línea, deserción escolar, retención estudiantil, factores personales, factores académicos. 


\title{
Influence of personal and academic factors on dropout of students in distance education
}

\begin{abstract}
Objective. Determine the elements that, according to the opinion of active students and deserters of this institution, are causing the problem of abandonment of the courses.

Method. The research had a quantitative approach of descriptive inferential, and yielded by applying a questionnaire some data on attitudes, trends, perceptions and opinions of participants regarding the influence of personal and academic factors, among others, which motivate them to stay in or drop out of courses in which they are enrolled.

Results. The main factors associated with dropout were: feeling of abandonment by tutors, academic factors, academic orientation, economics and family responsibilities, flexibility of space and time, among others.
\end{abstract}

Keywords: Distance education, online learning, college drop-out, student retention, personal factors, academic factors.

Recibido: 19-03-2015

Aceptado: 06-05-2015

\section{Introducción}

Si bien la educación a distancia ha sido un área de gran crecimiento en la educación a nivel internacional, ésta sufre una debilidad fundamental que es la alta tasa de deserción de las personas que estudian bajo esta modalidad (Boyle, Kwon, Ross \& Simpson, 2010). De acuerdo con Restrepo (2005), quien reportó el resultado de estudios realizados en diferentes contextos norteamericanos, europeos y latinoamericanos, las cifras de abandono en esta modalidad educativa, oscilan entre 40 y $70 \%$, tendencia confirmada en el estudio realizado en Colombia por el Ministerio de Educación Nacional, MEN, (2009), quien encontró para los programas a distancia una deserción superior al 60\%. De 
acuerdo con Inan, Yukselturk y Grant (2009), los valores de ausentismo escolar definitivo en la educación a distancia se encuentran entre 10 a $20 \%$ por encima de los de la educación convencional, afirmación que puede validarse con el hallazgo del MEN (2009), el cual presentó cifras del 48\% de deserción para programas presenciales.

Según Isaza (2009), la deserción se ha convertido en uno de los mayores costos sociales e individuales en la educación superior en Colombia. Esto concuerda con Nichols (2010), quien afirmó que uno de los mayores intereses tanto institucionales como del estudiante, es que este permanezca en su proceso educativo hasta finalizar el programa cursado, por los considerables costos que la deserción implica. Además de los costos financieros que afecta tanto a las instituciones como a los alumnos, existe un costo emocional para la persona que abandona sus estudios y es la ruptura de la confianza en sí mismo (Facundo, 2009). Velázquez-Couvertier (2013), encontró en su investigación sobre la percepción de los docentes acerca de la deserción escolar, que las repercusiones de esta última en la vida de sus actores son evidentes, especialmente en estos tiempos, donde el conocimiento es básico para el desarrollo individual, un eficiente desempeño profesional y el acceso a mejores oportunidades de vida y de trabajo.

Estos argumentos motivaron la realización de un estudio que permitiera identificar algunas razones de la deserción escolar en un sistema bajo la modalidad de educación superior a distancia en una universidad colombiana y hacer una propuesta de motivación y apoyo al estudiante, como posible medida para reducir el número de alumnos universitarios que no continúan sus estudios.

La investigación se realizó en la Universidad Nacional Abierta y a Distancia, institución educativa pública de carácter nacional, la cual tiene el mayor cubrimiento geográfico en la modalidad a distancia en Colombia, con programas que van desde bachillerato hasta posgrado. Su acción pedagógica está orientada hacia la formación integral del estudiante, con base en el aprendizaje autónomo, el estudio independiente y el acompañamiento tutorial.

Partiendo de la revisión de varios estudios sobre la deserción escolar en general, se encontró que entre las causas identificadas por diferentes autores sobre este problema, se resaltan factores del estudiante tales como preparación académica, personalidad, inteligencia, motivación y otros elementos externos como el ambiente institucional y el apoyo de la facultad. Estos planteamientos fueron confirmados por la Universidad de los Andes (2007) en una investigación realizada sobre las instituciones de educación superior en Colombia, donde encontró que la deserción estudiantil es el resultado de la interacción de diferentes factores: (a) individuales tales como: edad, género, expectativas personales y facilidades de integración social; (b) académicos como orientación profesional, rendimiento académico, metodología de estudio; (c) institucionales tales como 
calidad del programa, recursos de la institución, relaciones con profesores y los demás estudiantes; y (d) socioeconómicos como estrato social, situación laboral e ingresos.

El marco teórico sobre el cual se basó esta investigación fue el modelo de Rivera (2011), quien adecuó versiones anteriores de autores reconocidos en el tema, a la identificación de factores que inciden en la deserción de estudiantes a distancia. Este modelo agrupa en cinco tipos de variables, el fundamento de la decisión de desertar o permanecer: variables personales o de trasfondo como edad, género, estado civil, lugar de residencia, ingresos, entre otras; variables académicas como hábitos de estudio, apoyo académico, meta académica, disponibilidad de los cursos; variables ambientales como finanzas, horas de trabajo, estímulos externos, responsabilidades familiares y de trabajo; variables de integración social como el apoyo y la interacción de los compañeros y de la institución; y variables de apoyo técnico como manejo de plataforma, apoyo técnico y administrativo, y disponibilidad de materiales y actividades de aprendizaje. Este artículo hace referencia a los resultados relacionados con las variables personales y académicas.

\section{Metodología}

\section{Diseño}

El enfoque principal de esta investigación es de tipo cuantitativo, transversal por encuestas, ampliamente utilizado en educación por muchos años (Creswell, 2008). La aplicación de un cuestionario a las muestras seleccionadas, permitió describir y relacionar datos sobre actitudes, opiniones, conductas, tendencias y percepciones de los participantes, como factores que intervienen en su permanencia o deserción de los cursos a distancia. Este diseño facilitó la generalización de los datos a una población, a partir de la muestra seleccionada. Adicionalmente es importante aclarar que las preguntas de investigación planteadas, fueron resueltas mediante la evaluación de las variables de investigación.

\section{Participantes}

Con el objeto de responder a las preguntas de investigación planteadas, los participantes de esta investigación provinieron de tres de los 48 programas agrupados en las cinco escuelas con que cuenta la institución bajo estudio. Para ello se consideraron dos tipos de poblaciones: estudiantes activos y desertores. La población de estudiantes activos correspondió a 244 que en el momento de la investigación estaban tomando el curso de Termodinámica, debido a la facilidad de contacto de la investigadora con ellos, como tutora de este tópico. Considerando que dicho curso es visto por estudiantes de los programas de Ingeniería de Alimentos, Ingeniería Industrial e Ingeniería Ambiental y con el objeto de que las dos poblaciones fueran comparables, la población de desertores correspondió a 797 personas de los mismos programas, que abandonaron sus 
estudios durante el periodo 2010-1, de acuerdo con los datos proporcionados por la oficina de Registro y Control Académico de la institución donde se hizo el estudio.

Muestra de estudiantes activos. Se invitaron 244 estudiantes del curso de Termodinámica de los cuales respondieron 111, lo que corresponde a una tasa de respuesta de 45\%. Para el cálculo del tamaño de la muestra se realizó un muestreo sistemático, el cual de acuerdo con Scheaffer, Mendenhall y Lyman (2007) es ampliamente utilizado en un diseño de encuesta por muestreo, debido a que simplifica el proceso de determinación de la muestra. Este método se clasifica como un diseño mixto, ya que tiene características tanto de muestreo no aleatorio, como de aleatorio (Kumar, 2011).

A partir de esta aplicación se obtuvo un tamaño de muestra en 32 estudiantes con un límite para el error de estimación de 3 estudiantes. Para la selección de los participantes, teniendo en cuenta que la encuesta se ubicó en la Web y que los resultados se muestran en forma cronológica, de acuerdo con las entradas y respuestas de los estudiantes, se inició con el primer estudiante que respondió y luego se tomó cada estudiante ubicado 3 posiciones adelante. Así, se tomaron los estudiantes ubicados en las posiciones 1, 4, 7, 10, 13...etc., hasta cubrir la población total de los 111 que respondieron. Así se seleccionaron los 32 estudiantes, que fue la muestra de estudiantes activos con la que se trabajó.

Muestra de desertores. Se envió la invitación para participar en el estudio a 797 personas identificadas en la oficina de Registro y Control Académico, como desertores del año 2010, primer semestre, de las cuales respondieron 22 correspondiente a una tasa de respuesta de 3\%. Este último resultado se explica en el sentido de que como se esperaba, adicional al hecho de que varios correos rebotaron, se trata de personas que salieron de la institución y que difícilmente les interesa diligenciar un cuestionario enviado por ella. Por conveniencia de la investigadora se tomó el total de quienes contestaron, lo cual es posible en el caso de investigaciones por encuestas, cuando la población objetivo es pequeña (Creswell, 2008).

\section{Instrumentos}

Para recolectar la información se utilizó un cuestionario desarrollado por Rivera (2011), con el fin de investigar acerca de los factores que inciden en la retención o deserción del estudiante matriculado a distancia, en una universidad de Puerto Rico. De acuerdo con la autora del instrumento, este fue validado por un panel de tres expertos en las áreas de investigación, educación a distancia y retención de estudiantes universitarios; posteriormente sometido, revisado y aprobado por la Junta de Revisión Institucional (IRB) de la institución investigada y por la IRB de la universidad donde la autora obtuvo su título de doctorado. Adicionalmente fue sometido a la prueba de confiabilidad del alfa Cronbach, en la cual se obtuvo un valor de .932 para todas las variables, por lo que su grado de confiabilidad 
es alto. La investigadora en referencia autorizó la adaptación y utilización del instrumento, para cumplir con el propósito de este estudio.

El cuestionario utilizado consta de tres partes: en la primera se consideran aspectos demográficos, carga y promedio académico, y contempla las preguntas 1 a 12. La segunda, la cual abarca las preguntas 13 a 49, corresponde a 27 afirmaciones que se deben responder mediante una escala Likert, en consonancia con el grado de acuerdo o desacuerdo con el planteamiento presentado; se relaciona con consejería académica, meta académica, apoyo tecnológico, hábitos de estudio, disponibilidad de los cursos, carga académica, satisfacción, interacción y utilidad, entre otros. La tercera corresponde a dos preguntas abiertas, 50 y 51, que busca la opinión de los estudiantes, respecto a los factores que motivarían su permanencia o abandono de los cursos a distancia.

Este instrumento se adecuó a las condiciones del medio y del tiempo en el cual se aplicó, obteniéndose como resultado dos cuestionarios con los mismos ítems. Ambos instrumentos se dispusieron en la Web en forma electrónica, metodología que de acuerdo con Creswell (2008), se ha popularizado últimamente. El cuestionario para los estudiantes activos se ubicó en un sitio Web de fácil acceso para ellos, a través de los foros del curso de Termodinámica. El cuestionario para desertores igualmente se localizó en un sitio de la Web, y mediante un correo electrónico a esta población se les invitó y facilitó su acceso para su diligenciamiento. El contacto a través de email, permite llegar a una muestra de la población geográficamente dispersa, lo cual es conveniente para una recopilación de datos más rápida y económica (Creswell, 2008).

Las variables de la investigación, valoradas mediante los instrumentos, se establecieron con el fin de obtener indicadores, particularidades o características, que permitieran dar respuesta a las causas relacionadas con la deserción de los estudiantes a distancia, de la institución bajo estudio. En la Tabla 1 se presentan las preguntas relacionadas con las variables personales o de trasfondo, las cuales debían responderse marcando una opción de las proporcionadas. En la Tabla 2, se relacionan las preguntas referentes a las variables de interacción académica, que debían responderse mediante escala Likert. En paréntesis se muestra la opción correspondiente al cuestionario de los desertores. 
Tabla 1. Variables personales.

\begin{tabular}{|c|l|}
\hline 1 & Género \\
\hline 2 & Edad \\
\hline 3 & Estado Civil \\
\hline 4 & Tiempo dedicado al trabajo \\
\hline 5 & Lugar de residencia \\
\hline 6 & Ingreso individual mensual \\
\hline 7 & Promedio académico de bachillerato con que ingresó a la universidad \\
\hline 8 & Meta educativa (grado que espera(ba) obtener) \\
\hline 9 & Carga académica en número de créditos tomados por semestre \\
\hline 10 & Número de cursos a distancia que toma actualmente (que tomó en total) \\
\hline 11 & Promedio académico actual (alcanzado en general) \\
\hline 12 & Tipo de colegio de donde obtuvo el grado de bachiller \\
\hline
\end{tabular}

Fuente: Propia.

Tabla 2. Variables de interacción académica.

\begin{tabular}{|c|c|c|c|c|c|c|}
\hline & & $\begin{array}{l}\text { Totalmente } \\
\text { de Acuerdo }\end{array}$ & De acuerdo & Indeciso & $\begin{array}{c}\text { En } \\
\text { desacuerdo }\end{array}$ & $\begin{array}{l}\text { Totalmente } \\
\text { en } \\
\text { desacuerdo }\end{array}$ \\
\hline 1 & $\begin{array}{l}\text { Estudio(é) a distancia porque la } \\
\text { disponibilidad de los cursos se } \\
\text { ajusta(ba) a mis necesidades }\end{array}$ & & & & & \\
\hline 2 & $\begin{array}{l}\text { Los cursos a distancia me han } \\
\text { ayudado (ayudaron) a ser auto } \\
\text { disciplinado en mis estudios }\end{array}$ & & & & & \\
\hline 3 & $\begin{array}{l}\text { Los objetivos de los cursos } \\
\text { a distancia están claramente } \\
\text { definidos }\end{array}$ & & & & & \\
\hline 4 & $\begin{array}{c}\text { Me siento satisfecho(a) con } \\
\text { la calidad de los cursos a } \\
\text { distancia }\end{array}$ & & & & & \\
\hline 5 & $\begin{array}{c}\text { Los criterios de evaluación } \\
\text { en los cursos a distancia } \\
\text { se ajustan a los objetivos } \\
\text { propuestos }\end{array}$ & & & & & \\
\hline 6 & $\begin{array}{c}\text { Recibo(i) la orientación } \\
\text { académica necesaria para } \\
\text { continuar con mis estudios }\end{array}$ & & & & & \\
\hline 7 & $\begin{array}{c}\text { El curso a distancia } \\
\text { estimula(ba) mi capacidad } \\
\text { académica }\end{array}$ & & & & & \\
\hline
\end{tabular}

Fuente: Propia. 
Influencia de los factores personales y académicos sobre la deserción de estudiantes en la educación a distancia. Artículo producto de la investigación.

En la Tabla 3 se presentan las preguntas abiertas que conformaron la tercera parte de los cuestionarios, especificando las correspondientes a cada muestra. En las respuestas de los participantes se identificaron algunos elementos de importancia, relacionados con las variables personales y con las variables de interacción académica.

Tabla 3. Preguntas abiertas.

\begin{tabular}{|c|l|}
\hline \multirow{3}{*}{1} & $\begin{array}{l}\text { ¿Qué factores te motivan para permanecer en los cursos a distancia? } \\
\text { (Estudiantes activos) } \\
\text { ¿Qué factores te hubieran motivado para permanecer en los cursos a } \\
\text { distancia? (Desertores) }\end{array}$ \\
\hline \multirow{3}{*}{2} & $\begin{array}{l}\text { ¿Qué factores te motivarian para abandonar los cursos a distancia? } \\
\text { (Estudiantes activos) } \\
\text { ¿Qué factores te motivaron para abandonar los cursos a distancia? } \\
\text { (Desertores) }\end{array}$ \\
\hline
\end{tabular}

Fuente: Propia.

Para el análisis de los datos referentes a las variables personales, obtenidos mediante selección múltiple con única respuesta, se realizó una comparación de porcentajes de frecuencia entre estudiantes activos y desertores, a través diagramas de barras para algunos ítems y en forma descriptiva para otros.

Para las variables de interacción académica evaluadas mediante escala Likert, utilizando la estadística descriptiva se realizó el cálculo de la media muestral y de la varianza por ítem. Adicionalmente, con el fin de determinar si existe diferencia entre las apreciaciones de estudiantes activos y desertores, se utilizó la estadística inferencial: se aplicó el procedimiento de la Diferencia Mínima Significativa, LSD de Fisher (Least Significant Difference), basado en la prueba t de significación de diferencia de medias (Anderson, Sweeney y Williams, 2008). El valor de LSD obtenido se comparó con la diferencia del valor absoluto de las medias muestrales y para los casos donde dicha diferencia fue mayor que el valor de LSD, se rechazó la hipótesis nula.

Para las preguntas abiertas se realizó un análisis gráfico, con el fin de determinar la importancia que los participantes otorgan a las razones de retención y de abandono de los cursos.

\section{Resultados}

Con el fin de responder a las preguntas de investigación en relación con las variables bajo estudio, se realizó un análisis comparativo para los diferentes resultados.

\section{Variables personales o de trasfondo}

A continuación se presentan los datos encontrados para las dos muestras. 
Algunos de estos resultados se ilustran en forma gráfica y otros se mencionan en forma descriptiva.

Sobre el género, para el grupo de estudiantes activos se encontró que el 50\% de los participantes corresponde al sexo femenino y el 50\% al sexo masculino. En cuanto a la muestra de desertores el $23 \%$ pertenece al sexo femenino y el $77 \%$ al masculino.

En relación a la edad, en la Figura 1 se representan los rangos de edades de los participantes. Mientras que la mayoría de estudiantes activos son menores de 29 años, la mayoría de desertores están por encima de esta edad.

Figura 1. Rango de edad de los participantes.

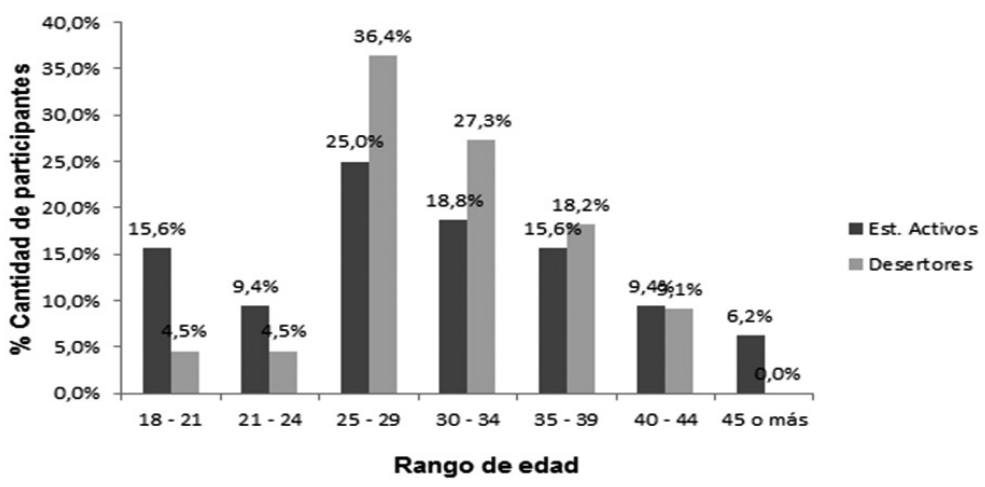

Fuente. Propia

Los datos de estado civil se representan en la Figura 2. Se observa que en la muestra de estudiantes activos la mayoría son solteros, en la de desertores la mayor parte son casados. Con respecto al lugar de residencia, se encontró que tanto la mayoría de estudiantes activos (56.3\%), como de desertores (50\%), residen en ciudades capitales.

Figura 2. Estado civil de los participantes.

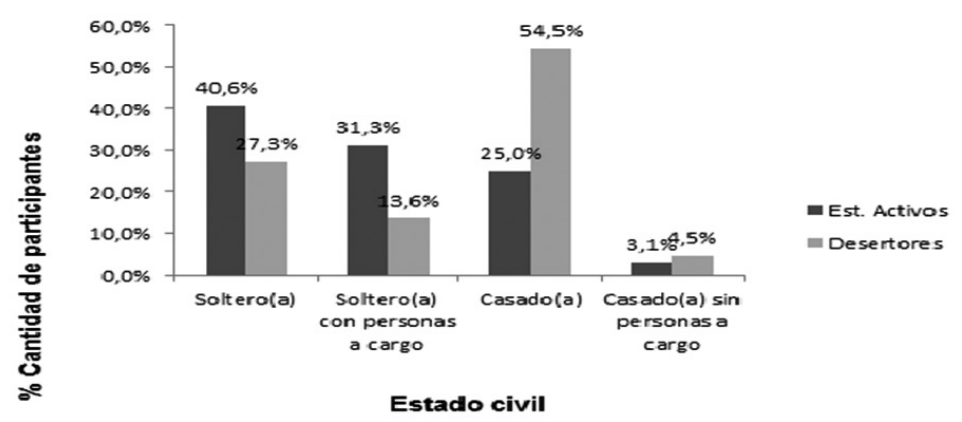

Fuente. Propia. 
Influencia de los factores personales y académicos sobre la deserción de estudiantes en la educación a distancia. Artículo producto de la investigación.

En la Figura 3 se ilustran los datos de salarios reportados. Se observa que la mayoría de estudiantes activos ganan entre cero y dos salarios mínimos, mientras que la mayoría de desertores ganan entre uno y cuatro salarios mínimos.

Figura 3. Ingreso individual mensual de los participantes.

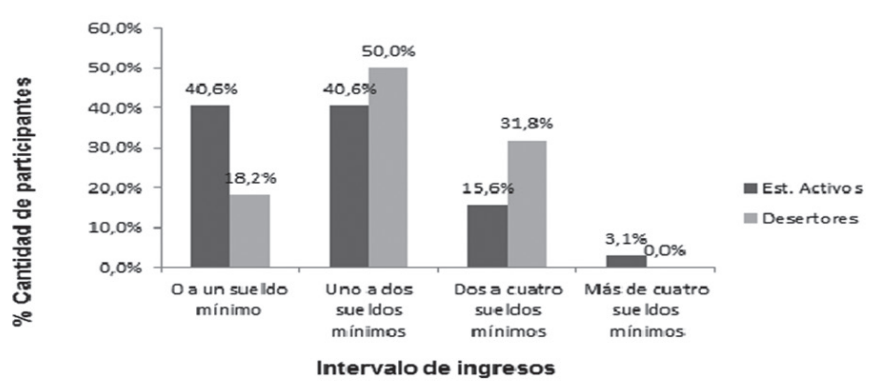

Fuente. Propia.

En la Figura 4 se representan los datos sobre el promedio académico obtenido en el bachillerato. En las dos muestras, la mayoría reportaron haber ingresado a la universidad con un promedio de 3.50 o más.

Figura 4. Promedio académico obtenido por los participantes en el bachillerato.

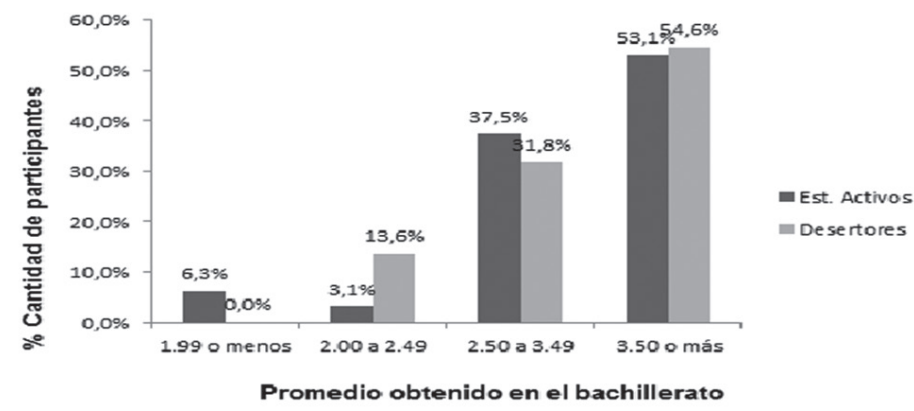

Fuente. Propia.

Con respecto al número de cursos a distancia tomados, se encontró que la mayoría de estudiantes activos están viendo cinco o más, mientras que la mayor parte de los desertores tomaron solo un curso. Estos resultados se ilustran en la Figura 5. 
Figura 5. Porcentaje de participantes por cantidad de cursos tomados a distancia.

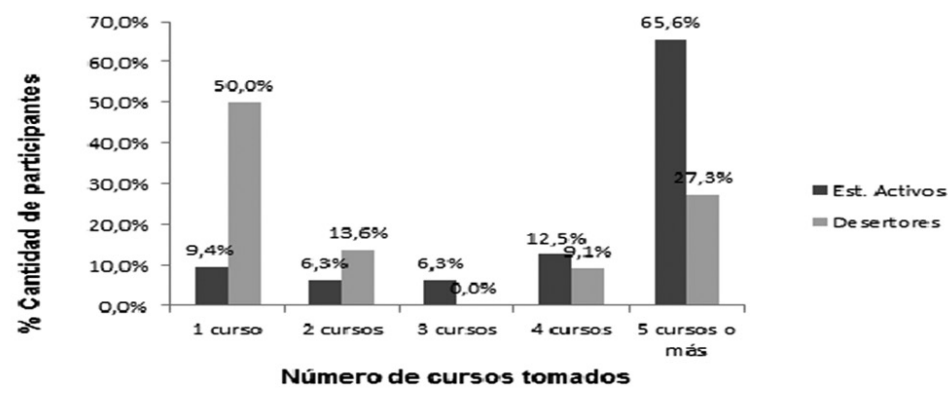

Fuente. Propia

Finalmente, en la pregunta sobre el tipo de colegio de donde obtuvieron su grado en el bachillerato, se encontró para la muestra de estudiantes activos que la gran mayoría, el $84.4 \%$, salieron de un colegio público. Por otro lado, el $54.6 \%$ de la muestra de desertores afirmaron ser egresados de instituciones públicas.

\section{Variables de interacción académica}

Para su análisis se consideraron las preguntas que tienen relación con la percepción de los estudiantes acerca del servicio y apoyo académico, calidad y disponibilidad de los cursos, y hábitos de estudio en cuanto a habilidades y destrezas, como orientadoras de la interacción académica. Estas fueron resueltas mediante escala Likert. En la Tabla 4 se muestran los valores de la media y la varianza para cada uno de estos ítems.

Tabla 4. Resultados de medias y varianzas de las variables de interacción académica.

\begin{tabular}{|c|c|c|c|c|c|}
\hline \multirow{2}{*}{$\mathrm{N}^{\circ}$} & \multirow{2}{*}{ Item } & \multicolumn{2}{|c|}{ Estudiantes Activos } & \multicolumn{2}{|c|}{ Desertores } \\
\hline & & Media & Varianza & Media & Varianza \\
\hline 14 & $\begin{array}{l}\text { Estudio(é) a distancia porque la disponibilidad de los } \\
\text { cursos se ajusta(ba) a mis necesidades }\end{array}$ & 4.469 & .49902 & 4.409 & .51446 \\
\hline 16 & $\begin{array}{l}\text { Los cursos a distancia me han ayudado (ayudaron) a } \\
\text { ser auto disciplinado en mis estudios }\end{array}$ & 4.406 & .61621 & 3.500 & 1.06818 \\
\hline 25 & $\begin{array}{c}\text { Los objetivos de los cursos a distancia están } \\
\text { claramente definidos }\end{array}$ & 4.031 & .78027 & 3.818 & .78512 \\
\hline 26 & $\begin{array}{c}\text { Me siento satisfecho(a) con la calidad de los cursos a } \\
\text { distancia }\end{array}$ & 3.469 & 1.12402 & 3.227 & 1.26653 \\
\hline 27 & $\begin{array}{c}\text { Los criterios de evaluación en los cursos a distancia se } \\
\text { ajustan a los objetivos propuestos }\end{array}$ & 3.469 & .99902 & 3.273 & 1.19835 \\
\hline 28 & $\begin{array}{l}\text { Recibo(i) la orientación académica necesaria para } \\
\text { continuar con mis estudios }\end{array}$ & 3.094 & 1.20996 & 2.682 & 1.76240 \\
\hline 48 & $\begin{array}{l}\text { El curso a distancia estimula(ba) mi capacidad } \\
\text { académica }\end{array}$ & 4.031 & .71777 & 3.818 & .87603 \\
\hline
\end{tabular}

Fuente: Propia 
Influencia de los factores personales y académicos sobre la deserción de estudiantes en la educación a distancia. Artículo producto de la investigación.

Adicionalmente, para las variables académicas, con el fin de determinar si existe diferencia entre las apreciaciones de estudiantes activos y desertores, se aplicó el procedimiento de la Diferencia Mínima Significativa, LSD. En la Tabla 5 se muestran los valores de LSD, la diferencia de medias y la comparación de dichos valores.

Tabla 5. Comparación de LSD y la diferencia absoluta de las medias para variables de interacción académica.

\begin{tabular}{|c|c|c|c|c|}
\hline $\mathrm{N}^{\circ}$ & Item & LSD & |Meac - Md| & Interpretación \\
\hline 14 & $\begin{array}{c}\text { Estudio(é) a distancia porque la disponibilidad de los } \\
\text { cursos se ajusta(ba) a mis necesidades }\end{array}$ & .40264 & .05975 & $L S D>|M e a c-M d|$ \\
\hline 16 & $\begin{array}{c}\text { Los cursos a distancia me han ayudado (ayudaron) a } \\
\text { ser auto disciplinado en mis estudios }\end{array}$ & .50675 & .90625 & $L S D<|M e a c-M d|$ \\
\hline 25 & $\begin{array}{c}\text { Los objetivos de los cursos a distancia están } \\
\text { claramente definidos }\end{array}$ & .61585 & .21307 & $L S D>|M e a c-M d|$ \\
\hline 26 & $\begin{array}{c}\text { Me siento satisfecho(a) con la calidad de los cursos a } \\
\text { distancia }\end{array}$ & .61585 & .24148 & $L S D>|M e a c-M d|$ \\
\hline 28 & $\begin{array}{c}\text { Los criterios de evaluación en los cursos a distancia se } \\
\text { ajustan a los objetivos propuestos }\end{array}$ & .58872 & .19602 & $L S D>|M e a c-M d|$ \\
\hline 48 & $\begin{array}{r}\text { Recibo(i) la orientación académica necesaria para } \\
\text { continuar con mis estudios }\end{array}$ & .67855 & .41193 & $L S D>|M e a c-M d|$ \\
\hline
\end{tabular}

Fuente: Propia.

Nota: $L S D=$ Diferencia Mínima Significativa con $d f=52$ y $p=.05 ;$ Meac $=$ Media estudiantes activos; $M d=$ Media desertores

\section{Discusión}

\section{Variables personales o de trasfondo.}

Los resultados sobre las variables personales o de trasfondo mostraron un comportamiento similar entre los estudiantes activos y los desertores, en la mayoría de las características estudiadas. Sin embargo, en los factores donde se evidenció alguna discrepancia entre las dos muestras de participantes, como fueron el género, el estado civil, la meta educativa y el número de cursos a distancia tomados, esta diferencia se puede explicar en la medida de que, para la muestra de desertores, a pesar de que se envió el correo a 797 personas, respondieron solamente 22 , la mayoría del sexo masculino que estaban matriculados en el programa de Ingeniería Industrial. La diferencia en el estado civil, cuyos datos mostraron que mientras en el grupo de estudiantes activos la mayoría son solteros, en la de desertores la mayor parte son casados, se entiende en la medida en que hay cuatro años de diferencia entre el tiempo en que los desertores estaban tomando los cursos (2010) y el tiempo actual, correspondiente a los estudiantes activos. 
De otro lado, revisando las respuestas a la pregunta abierta sobre las razones que los motivan a permanecer en los cursos, se encontraron como factores personales de importancia para aproximadamente la mitad de los estudiantes activos, la superación personal, familiar y laboral; y la necesidad de obtener un título. Estos resultados son consistentes con los hallazgos de Rivera (2011), quien en su investigación sobre los factores que inciden en la deserción o retención de estudiantes a distancia, encontró como causas de permanencia, entre otras, la alternativa de completar una carrera para mejorar su situación económica y de empleo, y poder cumplir con sus responsabilidades familiares. Estos resultados se entienden desde la perspectiva del ser humano, quien siempre está buscando mejorar su calidad de vida y la de su familia. En el caso de los desertores no se encontró razón de tipo personal que los hubiera motivado a permanecer en los cursos, lo que podría explicarse desde la visión de que, durante su corta estadía en la universidad fueron mayores sus razones de desmotivación que sus intereses personales de superación.

En relación con las respuestas a la pregunta abierta sobre las razones que los motivarían a abandonar los cursos, se encontró la referencia a la falta de tiempo, tanto por parte de los desertores como de los estudiantes activos, aunque en menor proporción. Este hallazgo confirma los encontrados por Facundo (2009), en su estudio de análisis sobre la deserción en la educación superior a distancia y virtual, realizado en la misma institución bajo estudio, y los de Gilbert (2000), quien observó como una de las razones más frecuentemente mencionadas en varias encuestas orientadas a buscar causas de deserción, la falta de tiempo. Similarmente Borges (2005), en su trabajo sobre la frustración del estudiante en línea, identificó la falta de tiempo debido a la desorganización personal, o atención a responsabilidades familiares y laborales, como una de las causas de desmotivación de los estudiantes. Además, la falta de organización es uno de los aspectos que frecuentemente se percibe en los programas a distancia, por lo que el tiempo que los estudiantes dedican a los cursos en muchas oportunidades es mínimo, conduciendo a la obtención de malos resultados y en consecuencia, de desmotivación y deserción académica.

Resumiendo, en esta investigación se encontró que, al parecer los factores demográficos no muestran mayor incidencia en las decisiones de abandonar o permanecer en los cursos, resultados que confirman los hallazgos de MenagerBeeley (2001), quien en su estudio sobre medir la motivación para identificar estudiantes en riesgo y mejorar la retención en las clases en línea, encontró que la mayoría de dichas características no parecen ser significativas en la conducta del estudiante y no influencian su persistencia en clase. De manera similar Park y Choi (2009), en su estudio realizado con el propósito de identificar los factores significativos que afectan la decisión del estudiante de desertar o persistir en el proceso de aprendizaje en línea, concluyeron que las características individuales de los aprendices, tales como edad, género y nivel educativo, no tienen un efecto significativo y directo sobre la decisión de desertar. 
Influencia de los factores personales y académicos sobre la deserción de estudiantes en la educación a distancia. Artículo producto de la investigación.

Por su parte Laskey y Hetzel (2011), en su investigación sobre los factores relacionados con la retención de estudiantes universitarios en riesgo de desertar, encontraron que los datos obtenidos no apoyaron la opinión de que las características demográficas y el perfil del colegio de donde salieron, afecten el proceso de permanencia de los estudiantes. Si bien en este estudio se encontró que existen algunas diferencias en cuanto a edad, estado civil, sexo y nivel de ocupación entre los estudiantes activos y los desertores, se pudo apreciar que estos factores demográficos no parecieran ser decisivos en el momento de desertar o permanecer en los cursos. Ahora bien, con respecto a los factores personales o de trasfondo, se pudieron identificar como razones de permanencia la necesidad de superación y de reconocimiento personal, mientras que las razones principales de abandono están relacionadas con la disponibilidad de tiempo.

\section{Variables de interacción académica}

Los resultados sobre las variables de interacción académica mostraron que son factores importantes en el momento de tomar la decisión de desertar o permanecer en los cursos. Para su interpretación se analizarán las apreciaciones de los estudiantes activos y de los desertores, con respecto a los cursos y al apoyo académico.

En relación con los cursos, vale la pena destacar entre los factores de permanencia señalados tanto por los estudiantes activos como por los desertores, su disponibilidad, la claridad de los objetivos y su capacidad para estimular las habilidades académicas, con lo cual las dos muestras se encuentran bastante satisfechas. Con respecto a la calidad de los cursos y los criterios de evaluación, a pesar de que los estudiantes activos manifestaron estar satisfechos y los desertores estar poco satisfechos, no se encontró una diferencia estadísticamente significativa entre sus apreciaciones.

Las respuestas a las preguntas abiertas, que mostraron alguna relación con los cursos, arrojaron como un factor importante de permanencia la flexibilidad de tiempo y espacio que estos les brindan, aspecto resaltado por una gran mayoría de los estudiantes activos. De otro lado, en una menor proporción los desertores manifestaron como una razón que los hubiera motivado a permanecer, una metodología más clara de los cursos, con mayor libertad de avance y menos trabajos colaborativos. Esto concuerda con sus respuestas sobre las razones que los motivaron a abandonar los cursos y con lo expuesto por algunos estudiantes activos, quienes abandonarían los cursos a distancia, por la metodología del trabajo colaborativo y un proceso de aprendizaje inadecuado.

Estos resultados confirman los encontrados por Rivera (2011), quien concluyó en su investigación realizada sobre los factores que inciden en la retención o deserción del estudiante matriculado a distancia, realizada en una universidad privada en el sur de Puerto Rico, que la mayoría de estudiantes permanecen en 
los cursos a distancia, por la disponibilidad y flexibilidad que estos ofrecen. Adicionalmente, de los resultados es evidente que la satisfacción con una buena metodología, con unos objetivos claros y con un aprendizaje significativo, es un factor básico para evitar la deserción. Al respecto Park y Choi (2009), encontraron que los aprendices están menos inclinados a desertar cuando están satisfechos con los cursos y cuando estos son relevantes para su vida. Por su parte Willging y Johnson (2009), reportaron como principal factor de influencia en la decisión de desertar o continuar, manifestado por estudiantes universitarios, el nivel de satisfacción con el primer o segundo curso en el programa. De estos hallazgos es claro identificar que la disponibilidad de los cursos, la capacidad de desarrollo académico del alumno y la flexibilidad de tiempo y espacio que éstos permiten, son razones muy importantes de permanencia identificadas tanto por los estudiantes activos como por los desertores bajo estudio en esta investigación.

Con respecto al factor relacionado con la orientación académica necesaria para continuar con los estudios, las dos muestras de participantes manifestaron estar poco satisfechas, lo cual permite percibir la baja interacción social referida al apoyo por parte del tutor. Los hallazgos sobre la falta de orientación o ausencia de una orientación académica eficiente, así como de la existencia de una metodología inadecuada en el proceso de enseñanza aprendizaje en la que se incluyen los trabajos colaborativos, se ubican entre las mayores causas de deserción identificadas en este estudio. Este resultado confirma el planteamiento de Gilbert (2000), quien encontró como conclusión de algunos estudios, que la naturaleza de los cursos a distancia, sus metas y métodos de instrucción, pueden ser definitivos en la decisión de su abandono.

Por otro lado, en la medida en que los estudiantes accedan a una buena orientación académica, se les facilita la comprensión de los cursos aumentando su retención en estos. En este mismo sentido, Laskey y Hetzel (2011), encontraron durante su estudio sobre factores relacionados con la retención de estudiantes en riesgo de desertar, que quienes mostraron una mayor tasa de retención fueron aquellos estudiantes que utilizaron los servicios de tutoría en una medida significativamente mayor. Es evidente entonces que el apoyo académico, o tutoría para esta institución, tiene un efecto positivo sobre la retención de los estudiantes.

En relación con el promedio académico se encontró que mientras la mayoría de los estudiantes activos tiene un promedio académico mayor de 2.5, tan solo un porcentaje cercano a la tercera parte de los desertores alcanzó dicha media durante su paso por la universidad. Este dato permite inferir que el bajo rendimiento académico es causa de deserción estudiantil, confirmando los hallazgos de Cabrera (2012), quien en su trabajo acerca de lecciones aprendidas sobre la persistencia y abandono de los estudios universitarios en los Estados Unidos, encontró que los estudiantes latinos tienen mayor probabilidad de permanecer en los cursos y graduarse, si mantienen un promedio académico 
igual o mayor a 2.5, sobre una escala de 1 a 4. Igualmente Schlenker (2006), en su estudio sobre desarrollo de un plan de retención para una pequeña universidad privada, encontró que el promedio académico es un fuerte indicador de persistencia y retención estudiantil en los cursos. Los resultados obtenidos en esta investigación, permiten considerar al bajo rendimiento académico, como una de las razones que motivaron a los desertores a abandonar los cursos.

\section{Conclusiones}

Los resultados de la investigación sobre los factores relacionados con las variables personales o de trasfondo y las variables de interacción académica, que inciden en la deserción de estudiantes en una universidad a distancia en Colombia, permitieron plantear las siguientes conclusiones:

1. Después del sentimiento de soledad y de abandono por parte de los tutores y de la institución, el factor de mayor incidencia en la decisión de desertar de los cursos, fue el relacionado con los factores académicos, percepción mostrada tanto por los estudiantes activos, como por los desertores. Estos hallazgos confirman los resultados obtenidos en la investigación de Rivera (2011), quien encontró como las variables más significativas en la deserción, los aspectos académicos y sociales.

2. La falta de una orientación académica clara y eficiente, y de una respuesta o realimentación oportuna, se encontraron entre las mayores causas de abandono de los cursos. Estos resultados confirman el planteamiento de Tinto (2012), quien manifestó que los estudiantes tienen una mayor probabilidad de éxito en ambientes que, entre otros, establecen expectativas claras de desempeño, proporcionan un buen apoyo académico, y evalúan y proporcionan realimentación oportuna de su desempeño.

3. Otros factores que vale la pena mencionar como razones de deserción manifestadas por los participantes en esta investigación, fueron la falta de tiempo, los aspectos económicos y las responsabilidades familiares. Estos aspectos son más responsabilidad de los estudiantes, quienes de acuerdo con Borges (2005), deberían prevenir, evitar y solventar las situaciones problemáticas relacionadas con estos y otros elementos personales, que les impidan un desempeño exitoso en su proceso de formación.

4. Como motivo de permanencia, el principal hallazgo fue la disponibilidad de los cursos, su capacidad de desarrollo académico y la flexibilidad de tiempo y espacio que estos permiten. Estos resultados confirman los encontrados por Willging y Johnson (2009), en su trabajo sobre los factores que influencian la decisión de los estudiantes para desertar de los cursos en línea, y los resultados de Rivera (2011), quien concluyó que la mayoría de estudiantes permanecen en los cursos a distancia, por su flexibilidad en términos de tiempo y disponibilidad. 
5. Entre los aspectos que no mostraron incidencia en la decisión de desertar, se encuentran los factores demográficos, coincidiendo estos hallazgos con los planteamientos de Menager-Beeley (2001), Park y Choi (2009) y Laskey y Hetzel (2011).

6. Finalmente, tanto la muestra de estudiantes activos como la de desertores mostraron insatisfacción con respecto a la orientación académica recibida, necesaria para su proceso de aprendizaje. En consecuencia se puede afirmar que la percepción de las dos muestras sobre las variables académicas relacionadas con el tutor y la institución, no presentan diferencias. Este resultado es un signo de alerta para la institución, en la medida de que el inconformismo sobre el proceso de orientación académica, es un sentimiento generalizado por todos los participantes.

\section{Referencias bibliográficas}

Anderson, D. Sweeney, D. Williams, T. 2008. Estadistica para administración y economía (10 $10^{\mathrm{a}}$ ed.). México, D.F.: Cengage Learning Editores, S.A.

Borges, F. 2005. La frustración del estudiante en línea. Causas y acciones preventivas. Digithum. Revista Electrónica de los Estudios de Humanidades y Filosofía de la UOC, 7. Disponible en: http://www.uoc.edu/digithum/7/dt/esp/borges.pdf

Boyle, F. Kwon, J. Ross, C. Simpson, O. 2010. Student-student mentoring for retention and engagement in distance education. Open Learning: The Journal of Open and Distance Learning, 25 (2): 115-130.

Cabrera, A. 2012. Lecciones aprendidas sobre el estudio de la persistencia y abandono de los estudios universitarios en los EEUU. Trabajo presentado en la II Conferencia Latinoamericana sobre el Abandono en la Educación Superior, Rio Grande do Sul. Resumen recuperado de http://www.education.umd.edu/Academics/Faculty/Bios/ facData/CHSE/cabrera/Lecciones_en_el_estudio_de_persistencia_en_los_EEUUv2. pdf

Creswell, W. 2008. Educational research: Planning, conducting and evaluating quantitative and qualitative research [Investigación educativa: planeación y evaluación de la investigación cuantitativa y cualitativa] (3a ed.). Upper Saddle River, NJ: Pearson Publishing.

Facundo, A. 2009. Análisis sobre la deserción en la educación superior a distancia y virtual: El caso de la UNAD - Colombia. Revista de Investigaciones UNAD, 8 (2): 117-149. 
Influencia de los factores personales y académicos sobre la deserción de estudiantes en la educación a distancia. Artículo producto de la investigación.

Gilbert, W. 2000. Retention in distance education telecourses and perceptions of faculty contact: A comparison of traditional and nontraditional community college students. (Tesis doctoral). Florida State University. USA

Inan, F. Yukselturk, E. Grant, M. 2009. Profiling potential dropout students by individual characteristics in an online certificate program. International Journal of Instructional Media, 36 (2): 163-172.

Isaza, J. 2009. Deserción en la Educación superior. El Observatorio de la Universidad Colombiana. Disponible en: http://www.universidad.edu.co/index.php?option=com content\&view=article\&id=689: desercion-en-la-educacion-superior\&catid=12:opini\& $\bar{l}$ temid $=200$

Kumar, R. 2011. Research methodology (3aed.). London: SAGE Publications Ltd.

Laskey, M. Hetzel, C. 2011. Investigating factors related to retention of at-risk college students. Learning Assistance Review, 16 (1): 31-43.

Menager-Beeley, R. 2001. Student success in web based distance learning: Measuring motivation to identify at risk students and improve retention in online classes. Presentación en línea. ( $\mathrm{N}^{\circ}$ de servicio de reproducción de documentos ERIC ED 466608).

Ministerio de Educación Nacional. 2009. Sistema de Prevención y Análisis de la Deserción en las Instituciones de Educación Superior en Colombia. Disponible en: http://www.mineducacion.gov.co/1621/article-156292.html

Nichols, M. 2010. Student perceptions of support services and the influence of targeted interventions on retention in distance education. Distance Education, 31 (1): 93-113.

Park, J. Choi, H. 2009. Factors influencing adult learners' decision to drop out or persist in online learning. Educational Teachnology \& Society, 12 (4): 207-217.

Restrepo, B. 2005. Consideraciones sobre el aseguramiento de la calidad en la educación virtual. Disponible en: http://www.colombiaaprende.edu.co/html/mediateca/1607/ articles-86323_archivo.pdf

Rivera, D. 2011. Factores que inciden en la retención o deserción del estudiante a distancia. (Tesis doctoral). Nova Southeastern University. USA.

Scheaffer, R. Mendenhall, W. Lyman, R. 2007. Elementos de muestreo (6aed.). Madrid: Thomson Editores

Schlenker, S. 2006. Development of a student retention plan for small private university with a plan for implementation an evaluation (Tesis doctoral). Nova Southeastern University. USA. 
Tinto, V. 2012. Completing collage: Rethinking institutional action. Chicago: University of Chicago Press.

Universidad de los Andes. 2007. Investigación sobre deserción en las instituciones de educación superior en Colombia. Disponible en: http://www.mineducacion.gov.co/ sistemasdeinformacion/1735/articles-254702_informe_tecnico_cede.pdf

Velázquez-Couvertier, D. 2013. La percepción de los docentes de un distrito escolar de Puerto Rico, sobre la deserción escolar y el programa desarrollo educativo general (GED) en la escuela superior (Tesis doctoral). Nova Southeastern University. USA.

Willging, P. Johnson, S. 2009. Factors that influence students' decision to dropout of online courses. Journal of Asynchronous Learning Nerworks, 13 (3): 115-127. 